\title{
Interaction layer between a turbulent boundary layer and free-stream turbulence
}

\author{
R. Jason Hearst, Eda Dogan \& Bharathram Ganapathisubramani
}

\begin{abstract}
The interaction between a turbulent free-stream and a turbulent boundary layer is investigated through particle image velocimetry measurements. An 'interaction layer' located between $0.12 \leq y / \delta \leq 0.19$, at the end of the log layer, is identified whereby the kinetic energy in this layer describes the flow above it. Conditional averages about the interaction layer indicate that it is home to peaks in the Reynolds stresses and that it is the location of a change in the vortical structure. Furthermore, the conditional information identifies that low kinetic energy deficit states in the interaction layer result in a more full boundary layer profile due to increased movement of the bulk flow towards the wall.
\end{abstract}

\section{Introduction}

The 'turbulent/non-turbulent interface' (TNTI) has been the subject of several recent investigations of the interaction between a turbulent boundary layer and an approximately laminar free-stream $[1,2,9]$. However, many engineering flows experience a turbulent free-stream. In such flows, one might expect there to be a 'turbulent/turbulent interface' (TTI) that distinguishes the turbulent boundary layer from the turbulent free-stream and where significant phenomenological interactions between these regions occur. As such, our focus here is to characterize these turbulent/turbulent interactions. This work differs from prior work examining the interaction of two different decaying turbulent free-streams (e.g., $[3,4])$ in that the boundary layer is a source of continuous production.

In the present study, we use an active grid to produce free-stream turbulence and measure the turbulent boundary layer on a flat plate, a setup inspired by Sharp et al. [10]. Using techniques pioneered for determining the TNTI between a canonical

R. Jason Hearst, Eda Dogan \& Bharathram Ganapathisubramani Engineering and the Environment, University of Southampton, SO17 1BJ, United Kingdom e-mail: r.j.hearst@southampton.ac.uk 
turbulent boundary layer and a laminar free-stream, we identify a layer-near the junction between the log and wake layers of the turbulent boundary layer-where significant interactions occur which are highly dependent on the local kinetic energy deficit.

\section{Experimental details}

An active grid, inspired by the original design of Makita [8], was used to generate free-stream turbulence. The active grid features a $11 \times 7$ array of square wings mounted to rods that are rotated in a random pattern by stepper motors to create a transient blockage. The grid has a mesh length, $M$, of $81 \mathrm{~mm}$. Changing the grid parameters and the free-stream velocity, $U_{\infty}$, allows for a variety of turbulence conditions to be produced. In the present study, we focus on one free-stream turbulence case in particular with the wings set to a rotational velocity of $\Omega=4.0 \mathrm{~Hz}$ with $U_{\infty}=8.1 \mathrm{~m} / \mathrm{s}$, resulting in $u^{\prime} / U_{\infty} \approx 12.2 \%$. For these conditions, the relevant freestream and boundary layer Reynolds numbers were $R e_{\lambda}=760$ and $R e_{\tau}=3329$, respectively.

Measurements were performed in the wake of the grid in a $0.9 \mathrm{~m} \times 0.6 \mathrm{~m} \times 4.5 \mathrm{~m}$ wind tunnel using planar particle image velocimetry (PIV) and hot-wire anemometry. The boundary layer was formed on a flat plate that begins $300 \mathrm{~mm}$ downstream of the grid and extends $4.2 \mathrm{~m}$ downstream. A single-wire, with a $1 \mathrm{~mm}$ sensing length, was located $3.5 \mathrm{~m}$ from the grid. It was operated with a Dantec StreamLine constant temperature anemometer at an overheat ratio of 1.8. The hot-wire was traversed in the wall-normal direction to measure a profile. Separately, PIV images were captured with three LaVision ImagerProLX CCD 16 mega-pixel cameras fitted with Nikon Nikkor $200 \mathrm{~mm}$ lenses. The light sheet was illuminated by a Litron Lasers Nano L200 15PIV Nd:YAG laser (523 nm wavelength, $200 \mathrm{~mJ} /$ pulse, $15 \mathrm{~Hz}$ repetition rate). The cameras were oriented to create a T-shaped field of view (FOV) extending $320 \mathrm{~mm}(\sim 2.6 \delta)$ in the streamwise direction and $268 \mathrm{~mm}(\sim 2.2 \delta)$ in the wall-normal direction after stitching the instantaneous images. The FOV begins $3240 \mathrm{~mm}$ downstream of the grid. The FOV was offset by $1.06 \mathrm{~mm}$ from the wall. A total of 2000 images were acquired. The boundary layer thickness was $\delta=\delta_{0.99}=122 \mathrm{~mm}$. In order to reduce the influence of pixel-locking, a Gaussian kernel filter was applied to the image pre-processing, window deformation was used during processing, and histogram equalization was applied on a vector-by-vector basis in post-processing [5]. 


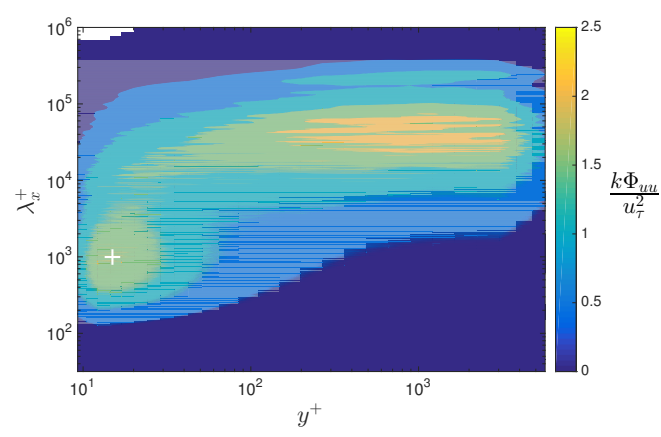

Fig. 1 Contour map of one-dimensional pre-multiplied spectra with wall-normal position. The $(+)$ marks the inner energy peak location identified by [6].

\section{Results}

\subsection{Wall-normal spectra}

The pre-multiplied energy spectra $\left(k \Phi_{u и}\right)$ measured by the hot-wire are briefly discussed here prior to investigating the PIV results. Contours of $k \Phi_{u u}$ for various wallnormal positions are shown in Figure 1. The spectra are plotted against wavelength, $\lambda_{x}$. Hutchins and Marusic [6] found that the near-wall peak in $k \Phi_{u u}$ in a canonical turbulent boundary layer did not significantly vary from $y^{+} \approx 15$ and $\lambda^{+} \approx 1000$ for $1010 \leq R e_{\tau} \leq 7300$, which is recovered for the present case despite significant free-stream turbulence. As Hutchins and Marusic [6] increased $R e_{\tau}$, they found that an outer peak in $k \Phi_{u u}$ emerged, and there was some indication this outer peak may be Reynolds number dependent. The present flow exhibits this outer peak, however, it is imposed by the free-stream turbulence. In essence, the near-wall spectra resemble those in a canonical turbulent boundary layer, while the $R e_{\tau}$ has been artificially increased by the imposition of the free-stream turbulence spectrum on the outer regions of the boundary layer.

\subsection{Interaction layer detection}

The instantaneous distribution of the local 2D turbulent kinetic energy deficit over a $3 \times 3$ area, may be estimated from $[1,2]$ :

$$
\tilde{k}=100 \times \frac{1}{9 U_{\infty}^{2}} \sum_{m, n=-1}^{1}\left[\left(\tilde{U}_{m, n}-U_{\infty}\right)^{2}+\tilde{V}_{m, n}^{2}\right]
$$




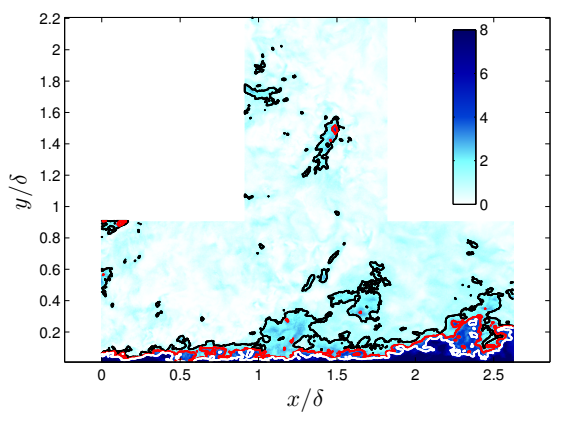

(a)

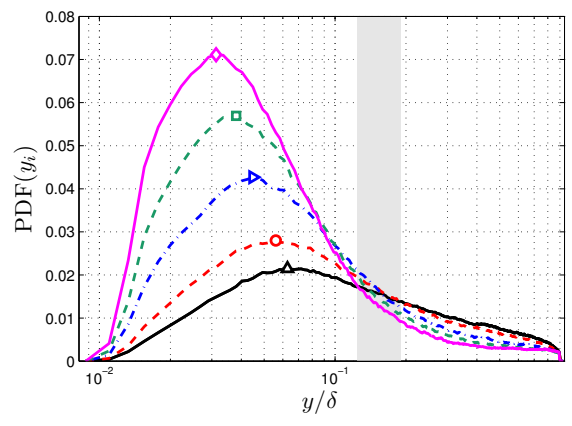

(b)

Fig. 2 (a) Representative image of instantaneous $\tilde{k}$ with contours at (black) $\frac{3}{2} k_{0}$, (red) $3 k_{0}$, and (white) $5 k_{0}$. (b) Probability density functions of the instantaneous wall-normal contour position with symbols marking peaks: $(\triangle) \frac{3}{2} k_{0},(\bigcirc) 2 k_{0},(\triangleright) 3 k_{0},(\square) 4 k_{0},(\diamond) 5 k_{0}$.

where $\tilde{U}_{m, n}$ and $\tilde{V}_{m, n}$ are the local streamwise and wall-normal velocities, respectively. When determining the TNTI, a threshold value, $k_{t h}$, is chosen that is representative of $k_{0}$ in the non-turbulent free-stream (typically, $k_{0}$ is near zero). At locations where $\tilde{k}>k_{t h}$, the flow is turbulent, and conversely $\tilde{k}<k_{t h}$ is non-turbulent. The contour drawn between the regions can be averaged over all images to determine the mean position of the TNTI (see [1] for more details). We employ a similar technique at several multiples of $k_{0}$, noting $k_{0} \gg 0$ in the present free-stream, to determine the various kinetic energy zones in the flow.

In Figure 2, contours at $k_{t h}=\frac{3}{2} k_{0}, 2 k_{0}, 3 k_{0}, 4 k_{0}$, and $5 k_{0}$, are presented. Figure 2(a) shows three of these contours on an instantaneous image, while Figure 2(b) shows the probability density functions (PDFs) composed over all images of the instantaneous wall-normal contour location, $y_{i}$. The symbols mark the PDF peak. The PDFs are non-Gaussian, and as such the mean of $y_{i}$ is not representative of the mostlikely position of the contour. This contrasts with the results for the TNTI where the $y_{i}$ distribution is Gaussian [1].

Of particular interest in Figure 2(b) is that all the PDFs cross within a narrow band of the boundary layer $(0.12 \leq y / \delta \leq 0.19)$, which is highlighted in Figure 2(b) and subsequent figures. This suggests that there is approximately the same likelihood of $\tilde{k}$ being at any state $\geq \frac{3}{2} k_{0}$ within this region. In Figure 3 the boundary layer profile is plotted in wall units with the canonical layers identified [7]. The 'interaction layer,' where the probability is the same that $\tilde{k}$ can take-on a wide range of values, occurs at the end of the log layer, prior to transition to the wake layer. 


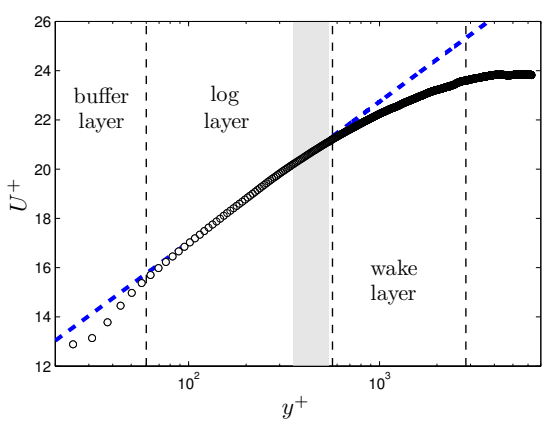

Fig. 3 Wall unit normalized mean velocity profile with log layer fit. Shaded area represents the interaction layer.

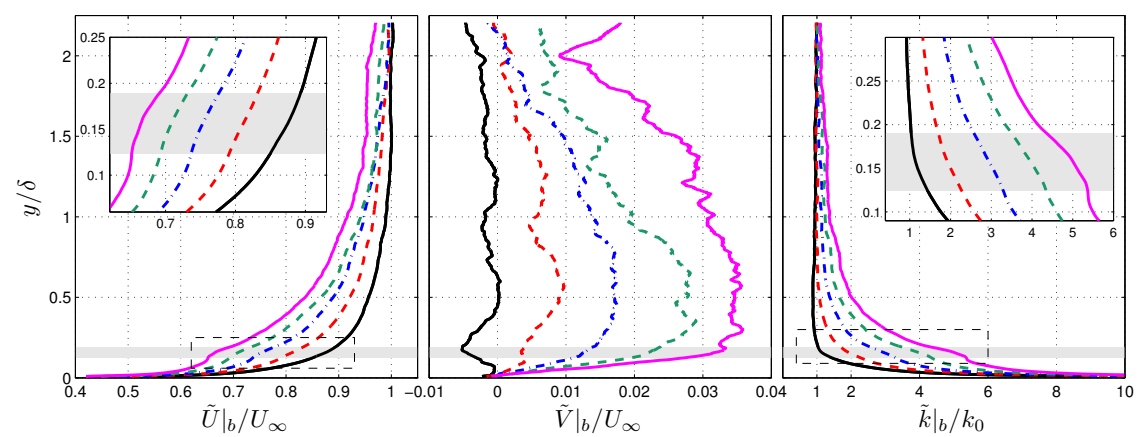

Fig. 4 Conditional averages of the local velocities and kinetic energy deficit. (一) $0.9 \leq \tilde{k}_{b} / k_{0}<$ $1.5,(--) 1.5 \leq \tilde{k}_{b} / k_{0}<2.5,(-\cdot-) 2.5 \leq \tilde{k}_{b} / k_{0}<3.5,(--) 3.5 \leq \tilde{k}_{b} / k_{0}<4.5$, (一) $4.5 \leq$ $\tilde{k}_{b} / k_{0}<5.5$.

\subsection{Conditional averages}

In order to ascertain the significance of the interaction layer, conditional averages were calculated for given ranges of the mean value of $\tilde{k}$ within the interaction layer; this quantity is denoted $\tilde{k}_{b}$, and a conditional average based on $\tilde{k}_{b}$ is denoted $\left.\cdot\right|_{b}$. The conditional averages of $\tilde{U}, \tilde{V}$, and $\tilde{k}$ are presented in Figure 4 . The value of $\tilde{k}_{b}$ distinctly influences the mean velocity profile to wall normal positions exceeding $\delta$. The boundary layer is the most 'full', i.e., has an extended region of high velocity close to the wall, for the instances with the lowest $\tilde{k}_{b}$. This is confirmed by the $\left.\tilde{V}\right|_{b}$ plot which reveals that the lowest kinetic energy deficit state draws the bulk flow towards the wall, whereas the other states push it away. Interestingly, the energy within the interaction layer appears to approximately set the values of $\tilde{U}$ and $\tilde{V}$ above it. 


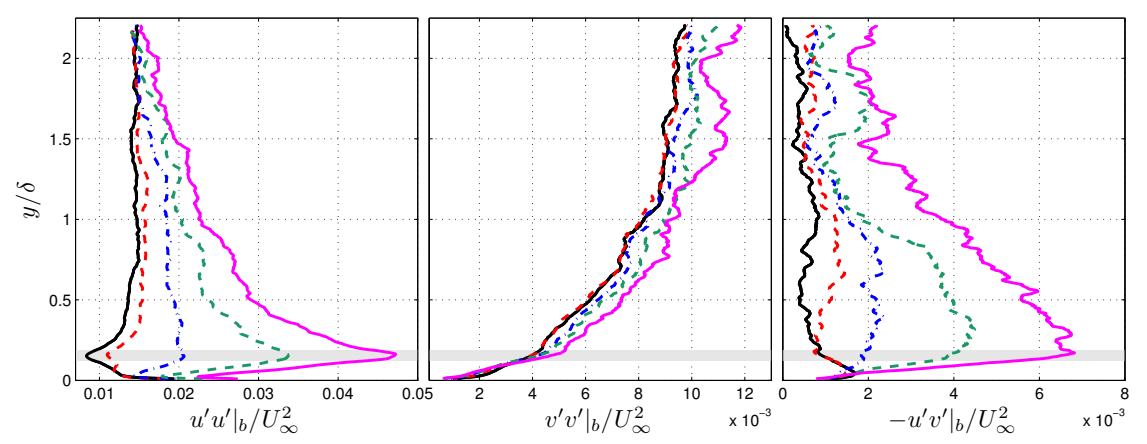

Fig. 5 Conditional averages of the local Reynolds stresses. (-) $0.9 \leq \tilde{k}_{b} / k_{0}<1.5$, (--) $1.5 \leq$ $\tilde{k}_{b} / k_{0}<2.5,(-\cdot-) 2.5 \leq \tilde{k}_{b} / k_{0}<3.5,(--) 3.5 \leq \tilde{k}_{b} / k_{0}<4.5,(-) 4.5 \leq \tilde{k}_{b} / k_{0}<5.5$.

In Figure 5 the conditional averages of the Reynolds stresses are shown. In particular, it is identified that the peak of $u^{\prime} u^{\prime}$ occurs in the interaction layer and scales with $\tilde{k}_{b}$. The near-wall peak of $u^{\prime} v^{\prime}$ scales with $\tilde{k}_{b}$, both in magnitude and in depth into the boundary layer. This is suggestive of locally higher skin-friction velocity, $u_{\tau}$, for the higher energy deficit states, which is consistent with the decreased boundary layer profile fullness for these same states shown in Figure 4.

\subsection{Vorticity and swirl}

The interaction between the free-stream and the boundary layer can also be accessed through the distribution of the vortices by observations of either the signed swirling strength or the vorticity. In the free-stream turbulence, one expects an equal number of vorticies rotating clockwise and counter-clockwise, while in the boundary layer dominated flow one expects a prevalence of clockwise rotating vorticies due to reduced velocity near the wall. In Figure 6(a) this principle is illustrated in a single snapshot, showing that indeed the near-wall flow is dominated by clockwise rotating vortices. The profiles of vorticity in Figure 6(b) indicate that near the wall the vorticity is negative signed, while as we move towards the free-stream the vorticity is equally distributed between positive and negative values resulting in a near zero mean. In the interaction layer, a local maximum and minimum follow each other in short succession for all cases save the lowest $\tilde{k}_{b}$. These peaks are separated by 30 vectors, which is highly suggestive that it is in fact in the flow and not an artefact of the resolution. From the wall to the interaction layer, the vorticity evolves monotonically, however, the local minimum near the top of the interaction layer appears to entrain fluid with positive vorticity to the bottom of the interaction layer where it is unable to penetrate further as the wall effects dominate. As such, the interaction layer may represent a barrier to intense vortical structures in the boundary layer. 


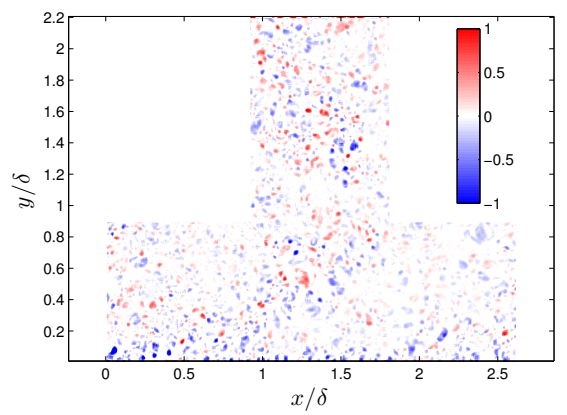

(a)

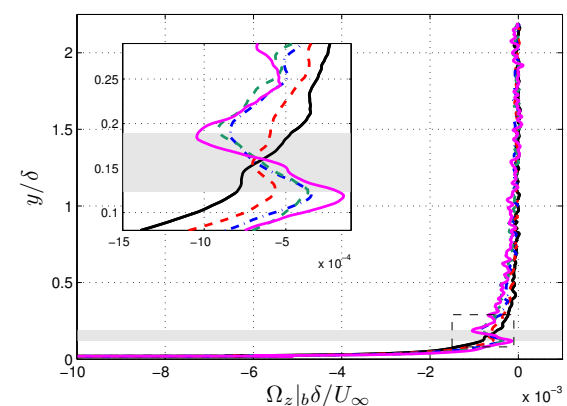

(b)

Fig. 6 (a) Representative image of instantaneous signed swirling strength. (b) Conditional averages of the transverse vorticity. (-) $0.9 \leq \tilde{k}_{b} / k_{0}<1.5,(--) 1.5 \leq \tilde{k}_{b} / k_{0}<2.5,(-\cdot-) 2.5 \leq \tilde{k}_{b} / k_{0}<$ $3.5,(--) 3.5 \leq \tilde{k}_{b} / k_{0}<4.5,(-) 4.5 \leq \tilde{k}_{b} / k_{0}<5.5$.

\section{Conclusions}

An interaction layer has been identified in a turbulent boundary layer with a turbulent free-stream. This layer exists in the range $0.12 \leq y / \delta \leq 0.19$ for the present flow and was investigated because the PDFs of the instantaneous kinetic energy deficits with different thresholds all took-on approximately the same value here. This region occurs at the end of the log layer and is home to the peaks in the Reynolds stresses as well as rapid changes in the vortical structure of the flow. It was also shown that for lower levels of the energy deficit near the wall, the boundary layer is more full. The low energy deficit state is accompanied by mean motion of the fluid towards the wall, which results in the more full profile.

Subsequent studies should focus on identifying clear criteria to instantaneously differentiate the turbulent boundary layer from the turbulent free-stream, if such a distinction is possible. Here, we have significantly identified a region of the flow that is home to energy dependent interactions and that may act as a barrier to the penetration of intense vortical structures. It was also demonstrated that the energy state within the interaction layer is representative of the flow structure above it, even exceeding $\delta_{0.99}$.

Acknowledgements We gratefully acknowledge the financial support of the European Research Council (ERC Grant agreement No. 277472) and EPSRC (Grant Ref No: EP/I037717/1), as well as the support of Dr. R. Hanson throughout the measurement campaign. RJH acknowledges the financial support of the Natural Sciences and Engineering Research Council of Canada (NSERC). 


\section{References}

1. Chauhan, K., Philip, J., de Silva, C.M., Hutchins, N., Marusic, I.: The turbulent/non-turbulent interface and entrainment in a boundary layer. J. Fluid Mech. 742, 119-151 (2014)

2. Chauhan, K., Philip, J., Marusic, I.: Scaling of the turbulent/non-turbulent interface in boundary layers. J. Fluid Mech. 751, 298-328 (2014)

3. Gerashchenko, S., Warhaft, Z.: Conditional entrainment statistics of inertial particles across shearless turbulent interfaces. Exp. Fluids 54(1631) (2013)

4. Good, G.H., Gerashchenko, S., Warhaft, Z.: Intermittency and inertial particle entrainment at a turbulent interface: the effect of the large-scale eddies. J. Fluid Mech. 694, 371-398 (2012)

5. Hearst, R.J., Ganapathisubramani, B.: Quantification and adjustment of pixel-locking in Particle Image Velocimetry. Exp. Fluids 56(191) (2015)

6. Hutchins, N., Marusic, I.: Evidence of very long meandering features in the logarithmic region of turbulent boundary layers. J. Fluid Mech. 579, 1-28 (2007)

7. Klewicki, J.C.: Reynolds number dependence, scaling, and dynamics of turbulent boundary layers. J. Fluids Eng. 132(094001) (2010)

8. Makita, H.: Realization of a large-scale turbulence field in a small wind tunnel. Fluid Dyn. Res. 8, 53-64 (1991)

9. Philip, J., Meneveau, C., de Silva, C.M., Marusic, I.: Multiscale analysis of fluxes at the turbulent/non-turbulent interface in high Reynolds number boundary layers. Phys. Fluids 26(015105) (2014)

10. Sharp, N., Neuscamman, S., Warhaft, Z.: Effects of large-scale free stream turbulence on a turbulent boundary layer. Phys. Fluids 21(095105) (2009) 